\title{
Dolor abdominal crónico, infestación por Blastocystis hominis, esofagitis y gastroenteritis eosinofílica
}

\section{Chronic abdominal pain, infestation by Blastocystis hominis, esophagitis and eosinophilic gastroenteritis}

Jaime Alfonso Ramírez-Mayans ${ }^{1}$, Karen Rubí Ignorosa-Arellano², Rodolfo Rodríguez-Jurado³

\section{RESUMEN DE LA HISTORIA CLINICA}

Paciente de 8 años, originario de Iztapalapa, Ciudad de México. Antecdentes heredofamiliares: madre de 28 años, con enfermedad de Kikuchi Fujimoto de 13 años, de evolución, sin complicaciones. Padre de 31 años sano. Hermano de 12 años, sano. Abuela paterna de 63 años con hipertiroidismo. Abuelo paterno que falleció por infarto agudo de miocardio y complicaciones de diabetes mellitus. Bisabuela materna con cáncer de mama, en tratamiento

Nació a término, Iloró y respiró al nacer, Apgar 8, peso 3100 g, longitud $52 \mathrm{~cm}$. Onfalorrexis a los 7 días. Crecimiento y desarrollo normales. Alimentación suficiente en cantidad y calidad. Inmunizaciones incompletas, BCG al nacer, HBV 2 dosis, pentavalente 3 dosis, Rotavirus una dosis, no había recibido la vacuna contra neumococo, refuerzo de DPT dos dosis, SPR una dosis; tamiz metabólico con resultado normal.

Antecedentes personales patológicos: fue hospitalizado en alrededor de cinco ocasiones por hiperreactividad bronquial, fue tratado con un disparo cada 12 horas de budesonida. La madre refiere que todo el tiempo tiene rinorrea hialina; tuvo varicela a los 3 años.

Padecimiento actual: siete meses de evolución, con dolor en el cuadrante superior izquierdo del abdomen, sin irradiación, intensidad 9/10, acompañado de náuseas, sin llegar al vómito, posprandial, inmediato, que se acompañaba de evacuaciones pastosas sin moco ni sangre. Estos

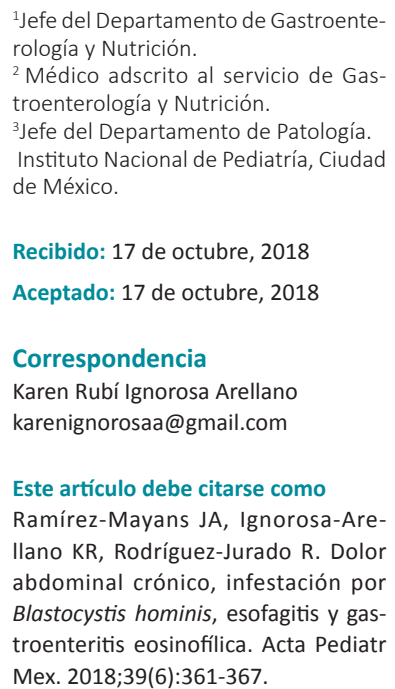

Este artículo debe citarse como Ramírez-Mayans JA, Ignorosa-AreIlano KR, Rodríguez-Jurado R. Dolor abdominal crónico, infestación por Blastocystis hominis, esofagitis y gastroenteritis eosinofílica. Acta Pediatr Mex. 2018;39(6):361-367. 
cuadros se presentaron dos veces por semana, sin relación con algún alimento. Cuatro meses más tarde, por exacerbación de los síntomas, fue Ilevado a Urgencias del Instituto Nacional de Pediatría. Le indicaron tratamiento con omeprazol, butilhioscina y trimebutina durante 14 días. Ante la falta de mejoría se solicitó una prueba de hidrogeniones, compatible con diagnóstico de intolerancia a la lactosa. Con este último tratamiento (reducción de la ingesta de lactosa) los síntomas disminuyeron. Las evacuaciones eran 2-3 veces por día (pastosas), según la escala de Bristol tipo 5, sin otros síntomas agregados.

Exploración física: peso $23.1 \mathrm{~kg}$ (10-25), talla 124 cm (10-25), IMC 15.02 (10-25). Edad aparente igual a la cronológica. Narinas permeables, con secreción mucosa hialina, faringe hiperémica, amígdalas con hipertrofia grado II, evidencia de exudado blanquecino. Caries grado II y III. Abdomen plano, peristalsis normoactiva, blando, depresible, sin visceromegalias palpables, sin datos de irritación peritoneal. A la percusión timpánica en el hemiabdomen inferior sin matidez hepatoesplénica. El resto de la exploración trascurrió sin anormalidades.

Valoración en el servicio de Gastro-nutrición: paciente con dolor abdominal crónico, con características de enfermedad ácido péptica, aunque también con datos de intolerancia a la lactosa. Se inició el protocolo para dolor abdominal, biometría hemática, pruebas de funcionamiento hepático, química sanguínea, tiempos de coagulación, proteína $\mathrm{C}$ reactiva, examen general de orina, coproparasitoscópico, inmunoglobulina E en suero (IgE), coprocultivo, química en heces, test de hidrogeniones y endoscopia.

\section{Endoscopia: estudio normal con hiato Hill I.}

Evolución (1 mes): sin disminución de los síntomas, dolor abdominal moderado a severo, intermitente con sensación de reflujo, evacuaciones disminuidas en consistencia, con moco, sin sangre, dos deposiciones al día acompañadas de distensión abdominal. Además, rinorrea continua acompañada de prurito nasal. A la exploración, sin dolor abdominal. Se inició tratamiento para infección por Blastocystis hominis con albendazol y secnidazol y luego budesonida intranasal, dieta estricta de restricción de lácteos.

Evolucionó con disminución de los síntomas. En la última consulta 2 meses después de haber iniciado el tratamiento refirió dolor abdominal intermitente asociado con falla en la dieta de exclusión.

\section{Comentario clínico de los doctores: Jaime A Ramírez Mayans y Karen R Ignorosa Arellano}

Se trató de un paciente que fue llevado a consulta por dolor abdominal crónico. Entre sus antecedentes importantes destacan los cuadros diarios de hiperreactividad bronquial y rinorrea hialina.

La semiología del dolor abdominal, a pesar de habérsele interrogado en forma adecuada, resultó inespecífica, por eso fue necesario solicitar exámenes de laboratorio y gabinete complementarios para poder identificar la causa.

Como datos positivos, en la biometría hemática se reportó eosinofilia, que puede orientar a datos de atopia o parasitosis. La IgE elevada y el examen coproparasitoscópico con Blastocystis hominis. La panendoscopia se reportó normal, sin evidencia de esofagitis o gastritis.

El dolor abdominal crónico en niños se define como, al menos, tres episodios de dolor en un lapso de 3 meses, que limitan la función, lo que corresponde al cuadro del paciente. Se desconoce la prevalencia mundial del dolor abdominal crónico en niños pero se estima que ocasiona de 5 a $10 \%$ de las visitas al pediatra. ${ }^{1,2}$ 
Si bien el síntoma dolor abdominal es un problema benigno en $90 \%$ de los casos, es causa de ausentismo escolar y de gran preocupación y angustia en los padres. ${ }^{3}$

La etiología se divide en causa funcional y orgánica. La primera se conoce en los casos donde no hay evidencia de alteraciones estructurales, bioquímicas, metabólicas, entre otras. El porcentaje varía, según la bibliografía, alrededor de $70 \%$ de casos funcionales. Las causas orgánicas se estiman en $30 \%$; las principales son infecciosas parasitarias (G. lamblia y B. hominis), esofagitis crónica, gastritis crónica, etc. ${ }^{1,2,3}$ En el Cuadro 1 se resumen las causas orgánicas y los datos clínicos predominantes para el diagnóstico diferencial en niños con dolor abdominal crónico. El hallazgo de $B$. hominis en el coproparasitoscópico obliga al tratamiento antiparasitario, como ocurrió en este caso. Sin embargo, el papel de este parásito como responsable de dolor abdominal aún es motivo de controversia. ${ }^{4,5}$ En el Cuadro 2 se muestran los datos de alarma a

Cuadro 1. Diagnóstico diferencial de dolor abdominal crónico en niños

\begin{tabular}{|c|c|}
\hline Causas & Síntomas \\
\hline $\begin{array}{l}\text { Infecciosas } \\
\text { Parásitos } \\
\text { (Giardia lamblia, } \\
\text { Blastocystis hominis, } \\
\text { Helicobacter pylori) }\end{array}$ & $\begin{array}{l}\text { Diarrea, borborigmos, disten- } \\
\text { sión abdominal, dolor abdo- } \\
\text { minal } \\
\text { Epigastralgia, saciedad tempra- } \\
\text { na, regurgitaciones }\end{array}$ \\
\hline $\begin{array}{l}\text { Malabsorción o intole- } \\
\text { rancia a carbohidratos, } \\
\text { lactosa, fructosa, } \\
\text { sorbitol, deficiencia de } \\
\text { sacarasa-isomaltasa }\end{array}$ & $\begin{array}{l}\text { Distensión abdominal, dolor } \\
\text { abdominal generalizado, gases, } \\
\text { diarrea osmótica }\end{array}$ \\
\hline $\begin{array}{l}\text { Inflamatorias } \\
\text { Esofagitis eosinofílica, } \\
\text { gastroenteritis eosinofí- } \\
\text { lica, enfermedad de } \\
\text { Crohn, colitis ulcerativa } \\
\text { enfermedad celiaca }\end{array}$ & $\begin{array}{l}\text { Diarrea, sangrado gastrointes- } \\
\text { tinal, pérdida de peso, dolor } \\
\text { abdominal }\end{array}$ \\
\hline $\begin{array}{l}\text { Síndrome de intestino } \\
\text { irritable }\end{array}$ & $\begin{array}{l}\text { Dolor abdominal asociado al } \\
\text { cambio de evacuaciones (fre- } \\
\text { cuencia, consistencia) }\end{array}$ \\
\hline
\end{tabular}

considerar en niños con dolor abdominal crónico. ${ }^{6,7}$ (Cuadros 1 y 2)

En el caso de este paciente en particular, el dato de hiperreactividad bronquial y rinitis de presentación frecuente, obligaron a pensar en una asociación del dolor abdominal crónico de tipo alérgico, en donde además de la causa alérgica se ha reportado, también, $B$. hominis. Ante el dolor abdominal con hiperreactividad bronquial siempre debe practicarse un estudio de $\mathrm{pH}$-impedanciometría porque la hiperreactividad bronquial puede ser explicada por el reflujo gastroesofágico. Otro diagnóstico a considerar es el de esofagitis eosinofílica, que es un padecimiento frecuente en el sexo masculino en pacientes con atopia: alergia alimentaria, asma, rinitis alérgica; los síntomas clínicos varían dependiendo del grupo de edad. ${ }^{8}$

En la endoscopia de este niño no hubo hallazgos sugerentes de esofagitis eosinofílica, como: traquealización esofágica, anillos circulares, atenuación del patrón vascular, pápulas blanquecinas y en algunos casos estenosis; ${ }^{8}$ por eso el diagnóstico solo puede documentarse por los hallazgos histopatológicos. Para establecer el

Cuadro 2. Reporte de estudios de laboratorio

\begin{tabular}{ll}
\hline BH & HB 13.3, HTO 39.7, VCM 88.6, CMH 29.6, \\
& LEU 13 100, N 6500, L 2300, M 500, E 900, \\
& PLA 366, 000 \\
\hline PFH & BT 0.49, BD 0.08, BI 0.41, AST 28, ALT 21, \\
& GGT 10, FA 231, PT 7.2, ALB 4.4, TG 57 \\
\hline QS & GLU 93, BUN 11.4, CREA 0.41 \\
\hline TC & TP 11 (103\%), INR 0.92, TTP 31.2 \\
\hline PCR & Negativo \\
\hline EGO & Amarillo, claro 1.020, pH negativo \\
\hline CPS & + Blastocystis hominis \\
\hline IGE & 205 IU/mL \\
\hline CPL & $\begin{array}{l}\text { Grasas en heces (+++), actividad tríptica (-), } \\
\text { azúcares reductores (+) }\end{array}$
\end{tabular}

Test de hidrogeniones $(\mathrm{H}+64$ ppm a las $2 \mathrm{~h})$. 
diagnóstico deben tomarse de 2 a 4 biopsias de los 3 tercios del esófago. ${ }^{8}$

Las infecciones parasitarias, como Giardia lamblia y $B$ hominis son causa de dolor abdominal crónico, y por el proceso inflamatorio generan intolerancia a la lactosa que puede agravar aún más el cuadro. En los últimos años se menciona que $B$ hominis se relaciona con padecimientos alérgicos, por lo que en este caso puede ser un factor implicado en los síntomas. ${ }^{4,5}$

Para el diagnóstico de intolerancia a la lactosa se requiere una prueba de hidrogeniones. Es positiva cuando la elevación es mayor de 20 partes por millón (ppm) de su medición basal a las 2 horas o síntomas gastrointestinales (dolor abdominal, vómito, diarrea). ${ }^{9}$ Esta prueba se le practicó al paciente y resultó positiva.

En este paciente, el dolor quizá era causado por la infestación por $B$. hominis, la esofagitis y gastroenteritis eosinofílica y la intolerancia a la lactosa.

Como se indica en este caso, el tratamiento es con un antiparasitario (secnidazol, albendazol, metronidazol) y restricción de lactosa durante 6 a 8 semanas.

\section{Hallazgos histopatológicos: Dr. Rodolfo Rodríguez Jurado}

Se recibieron como biopsias de esófago dos fragmentos pequeños de tejido que, en conjunto, midieron $0.3 \times 0.2 \times 0.2 \mathrm{~cm}$, marrón claro, de consistencia blanda. Los cortes histológicos mostraron epitelio esofágico con leve hiperplasia de la capa basal, con denso infiltrado inflamatorio eosinofílico; se contaron más de 25 eosinófilos por campo a seco fuerte (Figura 1). En el cardias también se encontró infiltrado eosinofílico (Figura 2). La biopsia del antro no mostró eosinofilia, solo edema leve. En el duodeno se encontró infiltrado eosinofílico moderado, hasta 12 eosinófilos por campo a seco fuerte. Figura 3

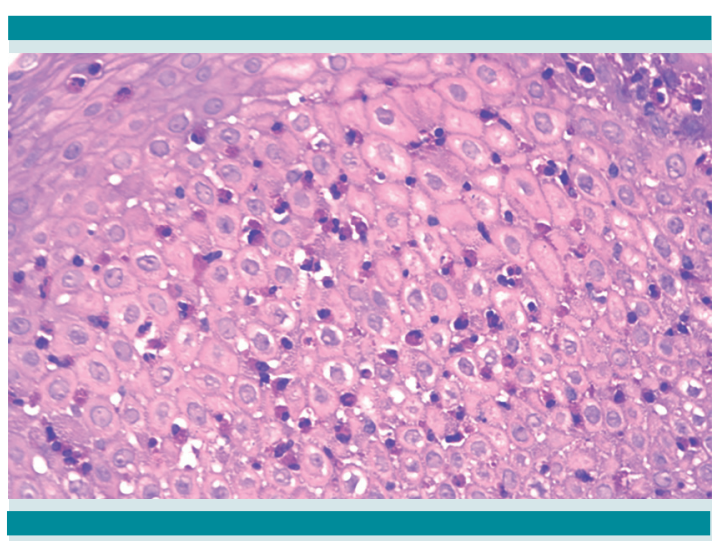

Figura 1. Epitelio esofágico con más de 25 eosinófilos en este campo a seco fuerte. Hematoxilina y eosina 40X

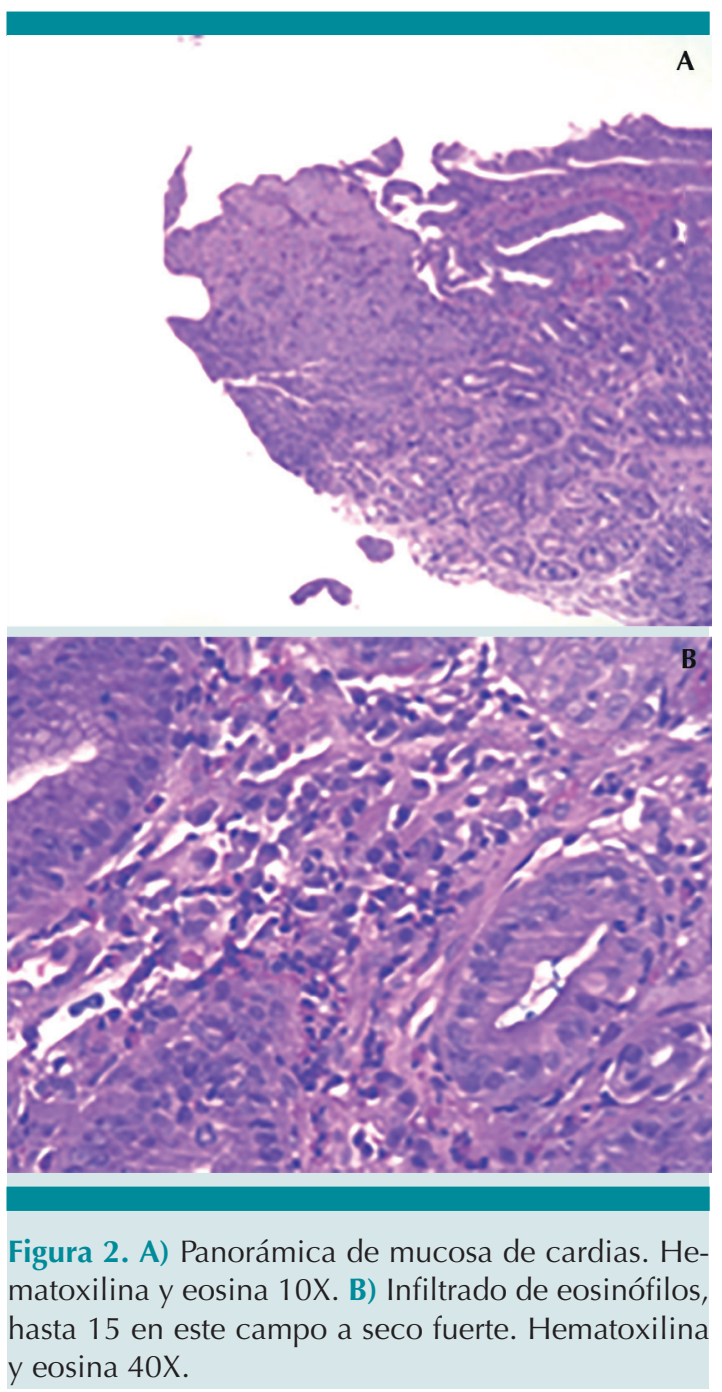




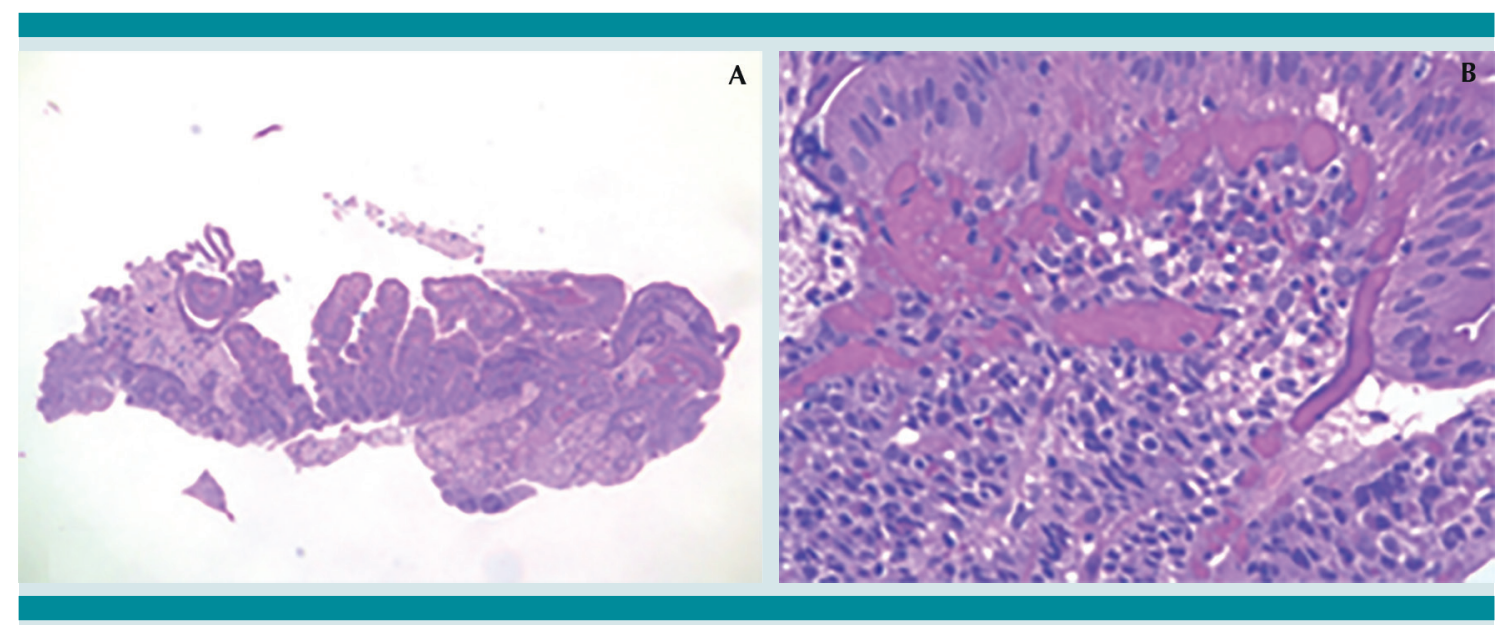

Figura 3. A) Panorámica de bulbo duodenal. Hematoxilina y eosina 10X. B) Vellosidad duodenal con infiltrado eosinofílico en la lámina propia. Hematoxilina y eosina 40X.

\section{Diagnósticos anatómicos}

1. Biopsias del esófago: esofagocarditis moderada, con eosinofilia acentuada.

2. Biopsias del antro y cuerpo gástrico: cambios reactivos inespecíficos.

3. Biopsias del bulbo duodenal: duodenitis crónica leve, no específica, con eosinofilia moderada.

Nota: la morfología sugiere trastorno gastrointestinal eosinofílico a descartar gastroenteritis eosinofílica versus esofagitis eosinofílica. La correlación clínica es necesaria.

\section{Comentario anatomopatológico}

La eosinofilia principal en este paciente fue en la mucosa esofágica. Las causas de esta eosinofilia fueron la esofagitis eosinofílica versus gastroenteritis eosinofílica. Por supuesto que deben descartarse otras causas, como la enfermedad por reflujo gastro-esofágico, eosinofilia esofágica respondedora a inhibidores de protones, enfermedad de Crohn, síndrome hipereosinofílico y enfermedades del tejido conectivo. ${ }^{10}$

La gastroenteritis eosinofílica en niños es una enfermedad heterogénea de patogénesis inmu- nológica. En algunos pacientes la enfermedad se relaciona con hipersensibilidad alimentaria, otros con parásitos, algunos con enfermedades vasculares del tejido conectivo y otros con etiología desconocida. ${ }^{11}$ Nuestro paciente tuvo Blastocystis hominis como factor implicado.

La forma común de la gastroenteritis eosinofílica es con edema en el antro, eritema y erosiones. En este padecimiento los menos afectados son el duodeno, esófago y cuerpo gástrico, en este orden.

Prusin considera que la gastroenteritis eosinofílica y la esofagitis eosinofílica son enfermedades estrechamente relacionadas. ${ }^{11}$ Comparten patogénesis común, inflamación tisular eosinofílica, enfermedad alérgica coexistente, eosinofilia periférica y sensibilización a alergenos alimentarios. Algunos pacientes con esofagitis eosinofílica tienen incremento de eosinófilos en el estómago y duodeno de significado no claro.

Gupta y su grupo ${ }^{12}$ encontraron que $12 \%$ de los pacientes pediátricos con esofagitis eosinofílica tienen eosinofilia gástrica, definida como más de 10 eosinófilos por campo a seco fuerte; no 
encontraron diferencias clínicas significativas entre los pacientes con esofagitis eosinofílica con o sin eosinofilia gástrica fortuita. Si nos atenemos a los límites duros de la cantidad de eosinófilos en el aparato digestivo de niños, la densidad de éstos en el duodeno está dentro de límites normales; es decir, menos de 30 eosinófilos por campo. ${ }^{13}$ Quizá sea necesario establecer nuestros propios límites normales de acuerdo con nuestra población.

\section{Comentario adicional}

La esofagitis y la gastroenteritis eosinofílica son formas únicas de alergia alimentaria no mediada por IgE; ambas se caracterizan por infiltración eosinofílica en niños y adolescentes. La evaluación de la eficacia de las restricciones dietéticas o las terapias farmacológicas para conseguir su curación clínica e histológica, mediante ensayos controlados con asignación al azar y metanálisis, ha dado lugar a nuevas directrices basadas en la evidencia. Evitar los desencadenantes de alimentos es el único tratamiento dirigido a la causa de la enfermedad. Ninguna de las pruebas de alergia alimentaria disponibles hoy en día predice adecuadamente los desencadenantes de alimentos para la esofagitis eosinofílica y gastroenteritis eosinofílica. Una dieta empírica de eliminación de alimentos sospechosos siempre ha proporcionado las mejores tasas de curación. En la actualidad, los inhibidores de la bomba de protones son el tratamiento de primera línea, se consigue una remisión histológica y disminución de los síntomas en 54.1 y $64.9 \%$, respectivamente. La eficacia de los corticoesteroides tópicos en esofagitis eosinofílica, evaluada en varios ensayos y resumida en metanálisis, indica que la budesonida y el propionato de fluticasona son significativamente superiores al placebo, en disminución de la infiltración de la mucosa eosinófila y en el alivio de los síntomas. Debido a las diferencias en el suministro de fármacos, las fórmulas de budesonida viscosa parecen ser la mejor opción farmacológica para la esofagitis eosinofílica.

Si la alergia a la proteína de la leche de vaca tiene un componente importante en este caso, la suspensión de leche y lácteos debería hacerse por un tiempo no menor a 6 meses. ${ }^{14}$

\section{REFERENCIAS}

1. Shulman RJ, et al. Characteristics of pain and stooling in children with recurrent abdominal pain. J Pediatr Gastroenterol Nutr. 2007; 44 (2): 203-8. doi: 10.1097/01. mpg.0000243437.39710.c0

2. Ramchandani PG, et al. The epidemiology of recurrent abdominal pain from 2 to 6 years of age: Results of a large population-based study. Pediatrics. 2005 ; 16(1): 46-50. doi: $10.1542 /$ peds.2004-1854

3. Sanz de la Garza CL, et al. Dolores abdominales recurrentes en atención primaria: estudio del dolor abdominal recurrente funcional. An Esp Pediatr. 2000; 53: 458-68. https:// doi.org/10.1016/S1695-4033(00)78629-2

4. Vichido Luna MA, et.al. Blastocystis hominis un agente patógeno controversial en la génesis de enfermedades gastrointestinales y alérgicas. Alergia, Asma e Inmunología Pediátricas. 2016; 25: 78-83.

5. Toro Monjaraz EM, et.al. Blastocystis Hominis and Chronic Abdominal Pain in Children: Is there an association between them? Journal of Tropical Pediatrics, 2018;64(4):27983. https://doi.org/10.1093/tropej/fmx060

6. Di Lorenzo C, et.al. Chronic abdominal pain in children: $A$ technical report of the American Academy of Pediatrics and the North American Society for Pediatric Gastroenterology, Hepatology and Nutrition. AAP Subcommittee and NASPGHAN Committee on Chronic Abdominal Pain. J Pediatr Gastroenterol Nutr. 2005; 40:249-61. doi: 10.1097/01. MPG.0000154661.39488.AC

7. Berger MY, et al. Chronic abdominal pain in children. BMJ 2007;334:997-1002. doi: https://doi.org/10.1136/ bmj.39189.465718.BE

8. Papadopoulou A, et.al. Management Guidelines of Eosinophilic Esophagitis in Childhood. Journal of Pediatric Gastroenterology and Nutrition. 2014; 58: 107-18.

9. Ignorosa AKR, et al. Absorción intestinal deficiente de lactosa; actualidades en pediatría. Alerg Asma Inmunol Pediatr. 2017; 26 (2): 49-55.

10. Ruchelli ED, Liacouras ChA. Esophageal Disorders in Childhood. In: Russo P, Ruchelli ED, Piccoli DA, eds. Pathology of Pediatric Gastrointestinal and Liver Disease. 2th ed. Berlin Heidelberg: Springer-Verlag, 2014;61-65. 
Ramírez-Mayans JA, et al. Dolor abdominal crónico y su relación con Blastocystis hominis

11. Prusin C. Eosinophilic gastroenteritis and related eosinophilic disorders. Gastroenterol Clin N Am. 2014;43: 317-327. doi: https://doi.org/10.1016/j.gtc.2014.02.013

12. Gupta SK, et al. Promising to indentify and monitor eosinophilic esophagitis. Clin Gastroenterol Hepatol 2017;15(11):1655-64. doi: 10.1016/j.cgh.2017.05.004.
13. Debrosse CW, et al. Quantity and distribution of eosinophils in the gastrointestinal tract of children. Pediatr Dev Pathol 2006;9:210-8. https://doi.org/10.2350/11-05-0130.1

14. Munoz-Persy M, et al. Treatment of eosinophilic esophagitis in the pediatric patient: an evidence-based approach. Eur J Pediatr. 2018;177:649-63. https://doi.org/10.1007/ s00431-018-3129-7

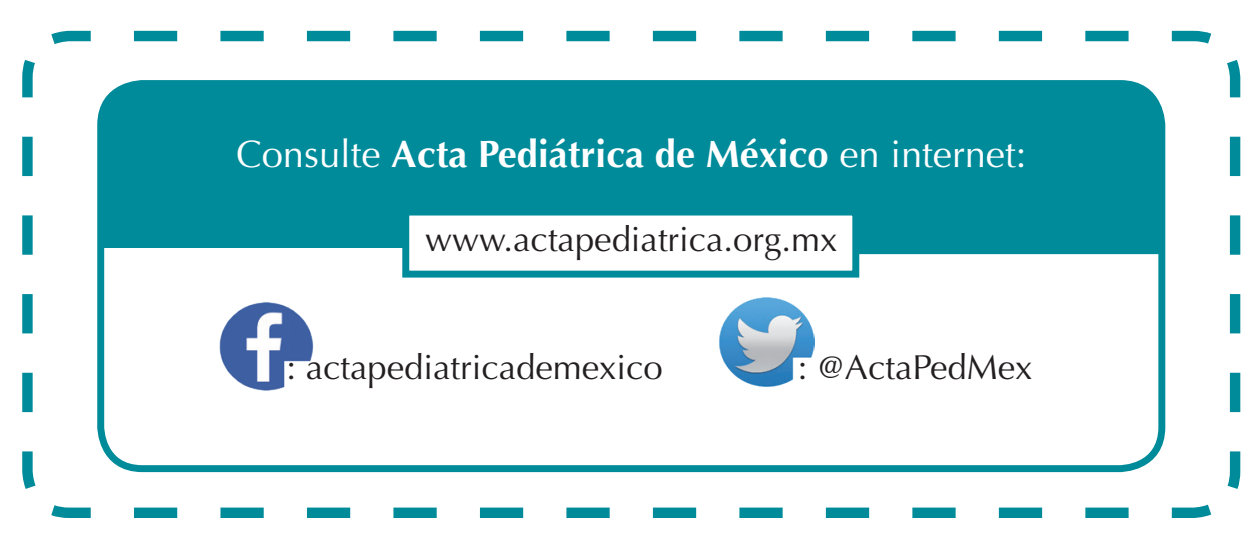

